\title{
A special issue on extensions of fuzzy sets in decision-making
}

\author{
Cengiz Kahraman ${ }^{1}$
}

Published online: 15 June 2018

๑) Springer-Verlag GmbH Germany, part of Springer Nature 2018

Decision-making is the study of identifying and choosing alternatives based on the preferences of decision-makers by taking the relevant evaluation criteria into account. Multiple criteria decision-making is a methodological tool for dealing with complex engineering problems. Multiple attribute decision-making refers to making selections among a finite number of decision alternatives in the presence of multiple, usually conflicting, attributes. In multiple objective decisionmaking problems, the number of alternatives is effectively infinite, and the trade-offs among the considered criteria are typically described by continuous functions.

Very often in MCDM problems, data are imprecise and fuzzy. Fuzzy set approaches are especially suitable when modeling human knowledge is required. Because of the need for better and detailed membership functions, ordinary fuzzy sets (Zadeh 1965) have been extended to type-2 fuzzy sets (Zadeh 1975), intuitionistic fuzzy sets (Atanassov 1986), fuzzy multisets (Yager 1996), neutrosophic sets (Smandarache 1999), nonstationary fuzzy sets (Garibaldi and Ozen 2007), hesitant fuzzy sets (Torra 2010), and Pythagorean fuzzy sets (Atanassov 1999; Yager 2013). Each of these extensions has been employed for developing the extensions of multiple criteria decision-making methods such as type-2 fuzzy TOPSIS, type-2 fuzzy AHP, intuitionistic fuzzy VIKOR, hesitant fuzzy ELECTRE, neutrosophic fuzzy EDAS, and Pythagorean fuzzy AHP. Intuitionistic fuzzy sets and hesitant fuzzy sets are the most used extensions of the ordinary fuzzy sets history. Pythagorean fuzzy sets and neutrosophic sets are the generalization of intuitionistic fuzzy sets, and they seem to be competitive with the other extensions of fuzzy sets in future.

This special issue aims to publish the best application and theoretical papers on decision-making using the extensions of ordinary fuzzy sets. Most of the papers have been selected from the presented papers at EUSFLAT 2017, September

Cengiz Kahraman

kahramanc@itu.edu.tr

1 Industrial Engineering Department, Istanbul Technical University, Istanbul, Turkey
11-15, 2017, Warsaw, Poland. The issue involves totally 18 papers after a peer review process.

The first paper presents the probabilistic ordered weighted averaging weighted average distance (POWAWAD) operator. POWAWAD is a new aggregation operator that uses distance measures in a unified framework between the probability, the weighted average, and the ordered weighted average (OWA) operator that considers the degree of importance that each concept has in the aggregation.

The second paper proposes a type-2 fuzzy multiple criteria methodology to evaluate and rank alternative waste collection systems in a smart city environment. Type-2 fuzzy TOPSIS method is applied to a real case study from Eskisehir, Turkey. Considering the current needs of Tepebaşı District, in which there are currently ongoing smart city projects funded by European Union, four alternative concepts, each is based on a particular information and communication technology, are designed.

The third paper extends EDAS method to its intervalvalued neutrosophic version with the advantage of considering an expert's truthiness, falsity, and indeterminacy, simultaneously. The proposed method is applied to the prioritization of United Nations national sustainable development goals, and one-at-a-time sensitivity analysis is conducted to check the robustness of the given decisions. The proposed method is also compared with the intuitionistic fuzzy TOPSIS method for its validity.

The fourth paper presents a new meta-heuristic algorithm, which is a new bio-inspired optimization algorithm based on the self-defense mechanisms of the plants. The proposed algorithm is based on the predator-prey model proposed by Lotka and Volterra, which means that when the plant detects the presence of an invading organism, it triggers a series of chemical reactions, which products are emitted into the air to attract the natural predator of the invading organism.

The fifth paper develops interval type- 2 fuzzy control charts. Interval type-2 fuzzy control charts can be obtained by using different methods such as defuzzification, centroid, type reduction, and likelihood. Comparisons are made between interval type-2 fuzzy control charts and classical 
control charts. It introduces likelihood method as a new approach to generate fuzzy control charts.

The sixth paper is on crucial and unsolved problems on Atanassov's intuitionistic fuzzy sets. Some researchers pointed out that some kinds of definitions of operations over Atanassov's intuitionistic fuzzy sets (IFSs) are incorrect. About 30 years after the first Atanassov's papers, there exist some misunderstandings related, not only on the name, but also on the basic operations on IFSs.

The seventh paper introduces a new Analytic Hierarchy Process (AHP) method with interval-valued neutrosophic sets. It also proposes an interval-valued neutrosophic AHP based on cosine similarity measures. The proposed method provides a scoring procedure for pairwise comparison matrices under neutrosophic uncertainty.

The eighth paper proposes some novel similarity measures to measure the relative strength of the different intuitionistic fuzzy sets after pointing out the weakness of the existing measures. The main component of the set pair analysis theory, a connection number, is formulated in the form of the degrees of identity, discrepancy, and contrary. Some new similarity and weighted similarity measures between the connection number sets are defined.

The ninth paper provides a novel retailer segmentation approach based on multi-criteria decision-making (MCDM)combined fuzzy data clustering. The proposed model consists of two phases: (1) an interval type-2 hesitant MCDM approach for the determination of location perceived value, (2) retailer (store) clustering via different product sale prices with fuzzy data-based fuzzy $\mathrm{C}$ means clustering. Using this integrated approach, advertisers and recommender system suppliers can manage their product-special offerings to customers considering retailer segments and shopping mall characteristics.

The tenth paper proposes a novel approach called Intuitionistic Cognitive Map (ICM) for assessing the criteria that influence the pricing strategy of a company in earlier stages of the product's life cycle in the market. A framework is formed based on a profound analysis of the literature and experts' opinions, in terms of criteria affecting pricing strategy and the causal relationships between them.

The eleventh paper applies the Pythagorean fuzzy set theory to incorporate the uncertainties into the economic analysis. The proposed Pythagorean Life-Cycle Cost (LCC) and Levelized Cost of Energy (LCOE) methods enable dealing with the solar energy investments with fuzzy parameters. Alternative energy systems with different technological features and economic conditions are compared by using the proposed method.

The twelfth paper focuses on the type- 2 fuzzy system design, proposing a multi-objective evolutionary approach for tuning the type- 2 inference engine of a fuzzy rule-based classification system by means of automatically choosing the t-norm used in the inference process. It proposes an approach to design and optimize type- 2 fuzzy systems that includes the tuning of type-2 fuzzy sets and the selection of rules.

The thirteenth paper develops a structure of interval type2 fuzzy p-control chart and interval type- 2 fuzzy np-control chart with constant sample size. The main advantage of using interval type-2 fuzzy sets in control charts is to provide the control of the flexibility on control limits while monitoring the process.

The fourteenth paper constructs the Haar measure on invariant state for fuzzy sets in a locally compact space. The invariant state is also studied on MV-algebra generated by a family of intuitionistic fuzzy sets.

The fifteenth paper studies how to define Szmidt \& Kacprzyk and Burrillo \& Bustince measures of Atanassov's IF entropies using measures of local divergence. In this context, it proves the necessary and sufficient conditions for defining entropy measures under both frameworks using local divergence measures for Atanassov's IF sets.

The sixteenth paper proposes an enhanced fuzzy evidential DEMATEL model to address inconsistent evaluations in emergency management systems. It uses Dempster-Shafer evidence theory to aggregate multiple opinions with the consideration of their corresponding total uncertainty measure. DEMATEL method is implemented to analyze the relationships among critical success factors.

The seventeenth paper identifies a set of relevant decision criteria and their sub-criteria needed in the evaluation of the Cloud Computing Technology (CCT) Provider Selection (PS) problem. It provides an integrated framework that can be used for the evaluation and selection of the most appropriate CCT provider, and then to apply this proposed approach in a real case study. The paper proposes a methodology consisting of IVIF AHP (Analytic Hierarchy Process), IVIF COPRAS (Complex Proportional Assessment), IVIF MULTIMOORA (Multi-objective Optimization by Ratio Analysis plus the Full Multiplicative Form), IVIF TOPSIS (Technique for Order of Preference by Similarity to Ideal Solution), and IVIF VIKOR (Višekriterijumsko kompromisno rangiranje) methods.

The eighteenth paper discusses the case that the elements of an index matrix are intuitionistic fuzzy pairs. In this case, it aggregates these elements by some intuitionistic fuzzy operations. A set of such operations is constructed so that the matrix elements are well ordered, generating a scale. Some applications of the so-constructed scaled operations are presented.

The nineteenth paper shows that assignment of an element to a class can be improved through alternative procedures more sensible to the available soft information. It proposes a general fuzzy bipolar approach that enables learning how to take advantage of the soft information provided by many 
classification algorithms in order to enhance the generalization power and accuracy of the classifiers.

We hope that this issue would provide a useful resource of ideas, techniques, and methods for present and further research on decision-making using fuzzy set extensions. My special thanks go to Prof. Vincenzo Loia, Editor Responsible for Special Issues, for his support and patience during the review process of the papers. I am also grateful to the referees whose valuable and highly appreciated works contributed to select the high-quality papers published in this issue.

Prof. Cengiz Kahraman Guest-Editor

\section{Compliance with ethical standards}

Conflict of interest The author declares that he has no conflict of interest.

Publisher's Note Springer Nature remains neutral with regard to jurisdictional claims in published maps and institutional affiliations. 